UTF-368/96

Jan 1996

\title{
Role of a "Local" Cosmological Constant in Euclidean Quantum Gravity.
}

\author{
Giovanni Modanese円 \\ I.N.F.N. - Gruppo Collegato di Trento \\ Dipartimento di Fisica dell'Universita' \\ I-38050 POVO (TN) - Italy
}

\begin{abstract}
In $4 \mathrm{D}$ non-perturbative Regge calculus a positive value of the effective cosmological constant characterizes the collapsed phase of the system. If a local term of the form $S^{\prime}=$ $\sum_{h \in\left\{h_{1}, h_{2}, \ldots\right\}} \lambda_{h} V_{h}$ is added to the gravitational action, where $\left\{h_{1}, h_{2}, \ldots\right\}$ is a subset of the hinges and $\left\{\lambda_{h}\right\}$ are positive constants, one expects that the volumes $V_{h_{1}}, V_{h_{2}}, \ldots$ tend to collapse and that the excitations of the lattice propagating through the hinges $\left\{h_{1}, h_{2}, \ldots\right\}$ are damped. We study the continuum analogue of this effect. The additional term $S^{\prime}$ may represent the coupling of the gravitational field to an external Bose condensate.

04.20.-q Classical general relativity.

04.60.-m Quantum gravity.
\end{abstract}

\footnotetext{
1e-mail: modanese@science.unitn.it
} 
Since the first perturbative formulations of quantum gravity it was realized that the addition of a cosmological term $\frac{\Lambda}{8 \pi G} \int d^{4} x \sqrt{g(x)}$ to the pure Einstein action gives the graviton a mass, which is positive if $\Lambda<0$ and negative - that means, the theory becomes unstable - if $\Lambda>0$ [1].

Nevertheless, whilst the vacuum fluctuations of the quantum fields should in principle produce a very large value of the cosmological constant, there is no observable hint of it. Namely, neither the Newtonian potential shows any finite range up to solar system distances, nor pure gravity exhibits any instability in the weak field case; finally the observations on cosmological scale set an upper limit on $|\Lambda|$ as small as $|\Lambda|<10^{-120} G^{-1}$ in natural units. This discrepancy is known as the "cosmological constant problem" and several possible escapes have been suggested [2]. Treating the problem at a fundamental level requires a non-perturbative approach, since one should be able to explain why the actual large-scale geometry of spacetime is flat just from dynamic considerations. It is therefore not surprising that a definitive and generally accepted solution of the paradox of the cosmological constant is still remote. We shall not make any attempt to a new explanation here.

We shall consider 4D pure gravity in the Euclidean approach, with special reference to quantum Regge calculus [3]. In this model the results of the numerical non-perturbative simulations compose the following picture of the behaviour of the cosmological constant: while its "bare" value $\lambda$ is generally nonzero, the effective value $\Lambda$ depends on the energy scale $\mu$ and vanishes at large distances like $|\Lambda| \sim G^{-1}\left(\mu l_{0}\right)^{\gamma}$, where $l_{0}$ is the lattice spacing and $\gamma$ a critical exponent. This means that the quantum geometry fluctuates on small scales, but reproduces flat space at macroscopic distances. The sign of $\Lambda$ is negative, thus the flat limit is well defined.

Our aim is to study the interaction of this gravitational system with a particular external source, namely a Bose condensate described by a scalar field $\phi(x)=\phi_{0}(x)+\tilde{\phi}(x)$. We assume that the vacuum density $\phi_{0}(x)$ is forced from the outside to a certain value, as it can happen for instance in a superconductor subjected to external electromagnetic fields. The positivity of the Euclidean action of $\phi$ ensures that the terms $\left[\partial^{\mu} \phi_{0}(x)\right]^{*}\left[\partial_{\mu} \phi_{0}(x)\right]$ and $m_{\phi}^{2}\left|\phi_{0}(x)\right|^{2}$ act like positive cosmological contributions to the gravitational action, possibly inducing local gravitational instabilities.

The structure of the paper is the following. In Section 1 we recall the main results of Euclidean 4D quantum Regge calculus concerning the effective cosmological constant. We 
mention its scale behaviour and two possible interpretations of the lattice spacing $l_{0}$. In Section 2 we consider in the continuum theory, at distances much larger than Planck scale, a weak Euclidean gravitational field $g_{\mu \nu}(x)=\delta_{\mu \nu}+\kappa h_{\mu \nu}(x)$, whose action includes an infinitesimal effective cosmological term with $\Lambda<0$. Such a theory can be regarded as the continuum limit of the lattice theory described in Section 1 . We then couple minimally $g_{\mu \nu}(x)$ to the mentioned scalar field $\phi(x)$, with vacuum density $\phi_{0}(x)$ determined from the outside, and write in detail

the various terms of the action. It turns out that the infinitesimal graviton mass $m_{g}^{2} \propto|\Lambda|$ receives a local negative contribution, which we denote by $-\mu^{2}(x)$. This means, as we show explicitly for the simpler case of an almost-massless scalar field $\chi$, that if there exist some fourdimensional regions in which $\mu^{2}(x)>m_{\chi}^{2}$, it is possible to find field configurations $\chi(x)$ which make the Euclidean action unbounded from below. The field tends to develope singularities in those regions, or - if a cut-off mechanism comes into play - it tends to assume constant extremal values which are independent from those in the neighboring regions. We suggest that these "constraints" should be inserted in the equation for the propagator of the field.

In Section 3 we remind the formula which gives the static potential energy in Euclidean quantum gravity as a functional average 4, 5]. We illustrate its statistical meaning in the case of a weak field through an analogy with a simple 2D Ising model, which we also treat numerically with an elementary simulation. We show numerically that the insertion of local supplementary constraints due to an external field (in analogy to the singularities of the continuum case) damps in a sensible way the spin-spin correlations, and thus the interaction they represent in the model.

\section{Regge calculus.}

In the last years discretized quantum gravity on the Regge lattice has led, through the Montecarlo numerical simulations of Hamber and Williams, to a better comprehension of the nonperturbative behaviour of the Euclidean gravitational field in four dimensions. Some of the features which emerge from their results [3] depend on the specific model, but several others are quite general. In this approach the physical quantities are extracted from functional 
averages. The partition function is written as

$$
Z=\int_{\text {Geometries }} d[g] e^{-\hbar^{-1} S[g]}
$$

where the geometries are described by piecewise flat simplicial manifolds. The integral (i.e. the Montecarlo sampling) runs over the lengths of the links which define a field configuration. The action in (11) has the form

$$
S=\int d^{4} x \sqrt{g}\left(\lambda-k R+\frac{1}{4} a R_{\mu \nu \rho \sigma} R^{\mu \nu \rho \sigma}\right)
$$

or, in discretized version,

$$
S[l]=\sum_{\text {hinges } h} V_{h}\left[\lambda-k \frac{A_{h} \delta_{h}}{V_{h}}+a \frac{A_{h}^{2} \delta_{h}^{2}}{V_{h}^{2}}\right]
$$

where $A_{h}$ is the area of an hinge, $V_{h}$ its volume and $\delta_{h}$ the defect angle (see the original papers for the definitions of lattice quantities and the functional measure).

In the following we shall set $\hbar=1$. The constants $k$ and $\lambda$ are related, as "bare" quantities, to the Newton constant $G$ and to the cosmological constant $\Lambda: k$ corresponds to $1 / 8 \pi G$ and $\lambda$ to $\Lambda / 8 \pi G$. It is important, however, to keep distinct the physical values $G$ and $\Lambda$ from $k$ and $\lambda$. The latter are entered as parameters at the beginning, and then a second order transition point for the statistical system described by $Z$ is found by Montecarlo simulation. Actually, there is a line of transition, since one can also vary the adimensional parameter $a$, which does not have a macroscopic counterpart. On this line in the parameter space the theory admits a continuum limit. Unlike in perturbation theory, where a flat background is introduced by hand, here the flat space appears dynamically; namely, the average value of the curvature is found to vanish on the transition line, which separates a "smooth phase", with small negative curvature, from a "rough", unphysical phase, with large positive curvature. In this way the effective, large scale cosmological constant

$$
\Lambda=\frac{\left\langle\int \sqrt{g} R\right\rangle}{\left\langle\int \sqrt{g}\right\rangle}
$$

vanishes in the continuum quantum theory.

More precisely, the dependence of the effective (or "running") cosmological constant $\Lambda_{\text {eff }}$ on the scale is the following. If we compute (4) on small volumes, the curvature fluctuates wildly. At larger distances the average curvature decreases, because the fluctuations tend to 
average out. If $\mu$ denotes the energy scale, close to the critical point the adimensional quantity $|\Lambda| G$ behaves like

$$
(|\Lambda| G)(\mu) \sim\left(l_{0} \mu\right)^{\gamma}
$$

where $l_{0}$ is the average spacing of the dynamical lattice and $\gamma \sim 1.56$ in the first simulations. The sign of $\Lambda$ is negative, as mentioned. Since the $\mu$-dependence of $G$ is quite weak, the effective cosmological constant decreases approximately like a power law as the length scale grows.

Eq. (5) admits two different physical interpretations, depending on the role we attribute to the average lattice spacing $l_{0}=\sqrt{\left\langle l^{2}\right\rangle}$. In the usual lattice theories, $l_{0}$ is sent to zero in order to obtain the continuum limit. In this first interpretation, eq. (5) shows the way the lattice theory reproduces flat space in the physical limit. No real physical meaning is assigned to the effective $\Lambda$ which, at a fixed scale $\mu$, is simply proportional to a positive power of the regulator.

On the other hand, we can believe that in quantum gravity $l_{0}$ has an intrinsic minimum value of the order of Planck length $L_{\text {Planck }} \sim 10^{-33} \mathrm{~cm}$. This hypotesis arises independently from several operational models (for a review see [6]) or from more complex quantum theories (see for instance [7] and references). In the framework of Regge calculus, it is possible to fix $l_{0}$ by imposing that the effective Newton constant $G$ computed non-perturbatively [5] is of the same magnitude order of the observed value; in this way one finds one more time that $l_{0}$ has to be of the order of Planck length [B].

In this second case, that is $l_{0} \sim L_{\text {Planck }}$, the interpretation of eq. (5) is different: it means that the effective cosmological constant tends to zero on large scale, while it is nonvanishing, in principle, on small scale (here and in the following we mean by "large" scale the laboratory or atomic scale, and by "small" scale the Planck scale). This interpretation does not necessarily have observable physical consequences, since in fact $\Lambda$ could be far too small. Namely, the exponent gamma has been computed only for small lattices; an evaluation for lattices of "macroscopic" size is of course technically impossible, and thus only the experiments could tell us whether the law (5) keeps true for large distances, and with which exponent. The fact that on astronomical scale we have $|\Lambda| G<10^{-120}$ for $\mu \sim 10^{-30} \mathrm{~cm}^{-1}$ constrains $\gamma$ to be approximately larger than 2. But the vanishing could be much more rapid, so that we could disregard $\Lambda$ at any physically relevant scale.

Another property of Regge calculus which shows an intrinsic feature of quantum gravity is the instability of the "rough" phase with positive average curvature, i.e. with positive effective 
cosmological constant. This phase does not admit any continuum limit. Its fractal dimension is small, which denotes that the geometry is collapsed.

The non-perturbative instability properties of the phase with positive average curvature extend the validity of known considerations based on perturbation theory. Namely, in the weak field approximation a positive cosmological constant in the gravitational lagrangian produces a negative mass for the graviton (compare [1] and the next Section). Instabilities arise in the perturbative theory on a De Sitter background too [9, 10]. On the contrary, a small negative cosmological constant generally does not imply any instability, but a small mass $m_{g}$ for the graviton, of the order of $\Lambda^{1 / 2}$ in natural units.

In conclusion, at a scale large enough with respect to the lattice scale it is possible to regard the discretized gravitational functional integral (1) as describing an almost flat mean field plus fluctuations. If the fixed point of the lattice theory is approached from the physical, smooth phase, the effective cosmological constant $\Lambda$ (i.e. the average of the scalar curvature $R$ ) is very small and negative, and the large scale fluctuations of $R$ are small too. The system is stable, because the field configurations with larger volume, in which the links are as stretched as possible, are preferred to the collapsed configurations, since the Euclidean action depends on the volume like $S \sim \frac{\Lambda}{8 \pi G} \int d^{4} x \sqrt{g} \sim \frac{\Lambda}{8 \pi G} \sum_{h} V_{h}$. (On the contrary, a positive value of $\Lambda$ would favour the collapsed configurations with smaller volume.) In this picture $\Lambda$ can be regarded either as a purely formal regulator, which goes to zero in the physical limit $l_{0} \rightarrow 0$, or as a physical quantity, though possibly extremely small (in the second interpretation, in which $\left.l_{0} \sim l_{\text {Planck }}\right)$.

Keeping these properties in mind, it is interesting to consider the case in which the coupling of the Euclidean gravitational field with an external source gives in some four-dimensional regions a positive contribution to the effective cosmological constant. In the next Section we shall analyse this phenomenon in the continuum case, that is, on large scale (in the meaning of "large scale" we precised above).

On the lattice, such a coupling would correspond in the action (3) to an additional term of the form

$$
\sum_{\left\{h_{1}, h_{2} \ldots\right\}} \lambda_{h} V_{h}
$$

where $\left\{h_{1}, h_{2} \ldots\right\}$ is a subset of the hinges and $\lambda_{h}$ are fixed positive constants. 
We expect that when the Montecarlo algorithm chooses for the random variation a link which ends in a hinge $h_{i} \epsilon\left\{h_{1}, h_{2} \ldots\right\}$, the favoured variation will be that for which the volume $V_{h_{i}}$ decreases. Thus the volumes $\left\{V_{h_{1}}, V_{h_{2}}, \ldots\right\}$ will tend to collapse and the lattice excitations propagating through the hinges $\left\{h_{1}, h_{2} \ldots\right\}$ will be damped.

\section{Continuum case.}

We have seen that the results of quantum Regge calculus can be interpreted as leading at distances large compared to $L_{\text {Planck }}$ to an effective Euclidean action for pure gravity of the form

$$
S_{e f f}=\int d^{4} x \sqrt{g(x)}\left[\frac{\Lambda}{8 \pi G}-\frac{1}{8 \pi G} R(x)\right]
$$

where the curvature fluctuations around flat space are small and the effective cosmological constant $\Lambda$ is negative and very small too. (As we saw in the previous Section, $\Lambda$ is scaledependent; we suppose here to stay at some fixed scale.) From the geometrical point of view, the small negative value of $\Lambda$ stabilizes the system, preventing it from falling into small-volume, collapsed configurations.

In the naive perturbation theory around the flat background the $\Lambda$-term represents a small mass for the graviton 2 . Namely, setting $g_{\mu \nu}(x)=\delta_{\mu \nu}+\kappa \tilde{h}_{\mu \nu}(x)$, with $\kappa=\sqrt{8 \pi G}$, the determinant $g$ of $g_{\mu \nu}$ can be expanded as

$$
g=1+\kappa \tilde{h}^{(1)}+\kappa^{2} \tilde{h}^{(2)}+\ldots
$$

where $\tilde{h}^{(1)}, \tilde{h}^{(2)}, \ldots$ denote expressions which are linear, quadratic etc. in $\tilde{h}_{\mu \nu}$. The linear "tadpole" term $\tilde{h}^{(1)}$ is usually disregarded, since it is proportional to the trace $\tilde{h}_{\mu}^{\mu}$, which vanishes on physical states. The term $\tilde{h}^{(2)}$ takes the form of a graviton mass term, such that the mass is positive when $\Lambda<0$ (compare [1]). We thus have

$$
\begin{gathered}
S_{\text {eff }}=\int d^{4} x\left\{\quad\left[m_{g}^{2} \tilde{h}^{(2)}(x)-\tilde{R}^{(2)}(x)\right]+\Lambda\left[\kappa \tilde{h}^{(3)}(x)+\kappa^{2} \tilde{h}^{(4)}(x)+\ldots\right]+\right. \\
\left.-\left[\kappa \tilde{R}^{(3)}(x)+\kappa^{2} \tilde{R}^{(4)}(x)+\ldots\right]\right\},
\end{gathered}
$$

\footnotetext{
${ }^{2}$ As remarked in [1], this widespread belief is not rigorously true. It has also been proved [11] that all theories of a massive tensor field in Minkowski space which satisfy the usual QFT postulates are incompatible with General Relativity in the limit of vanishing mass. In our reasoning it is not essential to regard the cosmological term as a graviton mass term, but for simplicity we stick to this terminology.
} 
where $m_{g}$ is proportional to $\Lambda^{1 / 2}$ and $\tilde{R}^{(2)}, \tilde{R}^{(3)} \ldots$ denote the parts of $R$ which are quadratic, cubic etc. in $\tilde{h}_{\mu \nu}$.

The first bracket contains the quadratic part of the action. The third bracket contains the familiar self-interaction vertices of the graviton, involving respectively $1,2, \ldots$ derivatives of $h_{\mu \nu}$. The second bracket contains self-interaction vertices which are peculiar of the theory with $\Lambda \neq 0$ and do not involve derivatives. We are however not interested in the self-interaction vertices of $h_{\mu \nu}$ in the following.

Now we would like to consider the interaction of $\tilde{h}$ with a scalar field $\phi$ having nonvanishing vacuum expectation value $\phi_{0}$. We suppose $\phi_{0}$ to be spacetime dependent and denote $\phi(x)=\phi_{0}(x)+\tilde{\phi}(x) ; \phi_{0}(x)$ is regarded as a quantity determined from the outside, that is, as a source term. In this way, the field $\phi(x)$ describes a Bose condensate with ground state density $\phi_{0}(x)$ fixed by external conditions (like, for instance, the Cooper pairs density in certain superconductors under external e.m. field).

The total action is

$$
\begin{aligned}
S=S_{\text {eff }}+\int d^{4} x \sqrt{g(x)} & \left\{\partial_{\mu}\left[\phi_{0}(x)+\tilde{\phi}(x)\right]^{*} \partial_{\nu}\left[\phi_{0}(x)+\tilde{\phi}(x)\right] g^{\mu \nu}(x)+\frac{1}{2} m_{\phi}^{2}\left|\phi_{0}(x)\right|^{2}\right. \\
& \left.+\frac{1}{2} m_{\phi}^{2}\left[\phi_{0}^{*}(x) \tilde{\phi}(x)+\phi_{0}(x) \tilde{\phi}^{*}(x)\right]+\frac{1}{2} m^{2}|\tilde{\phi}(x)|^{2}\right\}
\end{aligned}
$$

We can rewrite it as

$$
S=\int d^{4} x \sqrt{g(x)}\left\{\left[\frac{\Lambda}{8 \pi G}+\frac{1}{2} \mu^{2}(x)\right]-\frac{1}{8 \pi G} R(x)\right\}+S_{1}+S_{2},
$$

where

$$
\begin{aligned}
\frac{1}{2} \mu^{2}(x)= & \frac{1}{2}\left[\partial_{\mu} \phi_{0}^{*}(x)\right]\left[\partial^{\mu} \phi_{0}(x)\right]+\frac{1}{2} m_{\phi}^{2}\left|\phi_{0}(x)\right|^{2} ; \\
S_{1}= & \frac{1}{2} \int d^{4} x \sqrt{g(x)} \partial_{\mu} \phi_{0}^{*}(x) \partial_{\nu} \phi_{0}(x) \kappa \tilde{h}^{\mu \nu}(x) \\
S_{2}= & \frac{1}{2} \int d^{4} x \sqrt{g(x)}\left\{m_{\phi}^{2}|\tilde{\phi}(x)|^{2}+m_{\phi}^{2}\left[\phi_{0}^{*}(x) \tilde{\phi}(x)+\phi_{0}(x) \tilde{\phi}^{*}(x)\right]+\right. \\
& \left.+\left[\partial_{\mu} \tilde{\phi}^{*}(x) \partial_{\nu} \tilde{\phi}(x)+\partial_{\mu} \phi_{0}^{*}(x) \partial_{\nu} \tilde{\phi}(x)+\partial_{\mu} \tilde{\phi}^{*}(x) \partial_{\nu} \phi_{0}(x)\right] g^{\mu \nu}(x)\right\}
\end{aligned}
$$

For brevity, we have not expanded here $\sqrt{g}$ and $R$ like in eq. (9). Let us first look at the terms $S_{1}$ and $S_{2}$. The term $S_{1}$ describes a process in which gravitons are produced by the source $\phi_{0}(x)$. The term $S_{2}$ contains the free action of the field $\tilde{\phi}(x)$, which describes the excitations of the condensate, and several vertices in which the graviton field $\tilde{h}_{\mu \nu}(x)$ and $\tilde{\phi}(x)$ 
interact between themselves and possibly with the source. All these interactions are not of special interest here and are generally very weak, due to the smallness of the coupling $\kappa$. The relevant point is that the purely gravitational cosmological term $\frac{\Lambda}{8 \pi G}$ receives a "local" positive contribution $\frac{1}{2} \mu^{2}(x)$ which depends on the fixed external source $\phi_{0}(x)$. According to eq.s (9), (7) and to our discussion of the sign of $\Lambda$, this amounts to a negative mass contribution and could lead to instabilities.

Let us study the effect of such a local negative mass contribution in the simpler case of a scalar field in flat space. We consider a scalar field $\chi$ with very small mass $m_{\chi}$ and add to its free Euclidean action a source term of the form $-\frac{1}{2} \mu^{2}(x) \chi^{2}(x)$, which represents a "localized negative mass". The action becomes

$$
S_{\chi}=\frac{1}{2} \int d^{4} x\left\{\left[\partial^{\mu} \chi(x)\right]\left[\partial_{\mu} \chi(x)\right]+m_{\chi}^{2} \chi^{2}(x)-\mu^{2}(x) \chi^{2}(x)\right\}
$$

To fix the ideas, let us suppose that $\mu^{2}(x)$ is different from zero in certain four-dimensional regions $\Omega_{i}$, where it takes the constant values $\mu_{i}$. Outside these regions let $\mu^{2}(x)$ go rapidly to zero. The solution of the classical field equation for $\chi$ is obtained by minimizing the action. In the absence of the source term we would have of course $\chi(x)=$ const. $=0$, because this minimizes at the same time the gradient term $\frac{1}{2}\left[\partial^{\mu} \chi(x) \partial_{\mu} \chi(x)\right]$ and the mass term $\frac{1}{2} m_{\chi}^{2} \chi^{2}(x)$ (both positive-defined).

In the presence of the source term $-\frac{1}{2} \mu^{2}(x) \chi^{2}(x)$, the action decreases when $\chi^{2}$ becomes large within the regions $\Omega_{i}$. The growth of $\chi$ in these regions is limited only by the fact that outside them $\chi$ must go to zero (due to the term $m_{\chi}^{2}$ ) and that the gradient in the transition region cannot in turn be too large. Let us suppose for instance that there is only one region $\Omega_{0}$, with the shape of a 4 -sphere of radius $r_{0}$. Let $\mu(x)$ take the value $\mu_{0}$ inside $\Omega_{0}$ and zero outside. We consider for $\chi$ the trial function $\chi(r)=\chi_{0} f(r)$. The action becomes (we admit spherical symmetry)

$$
S_{\chi}=\pi^{2} \chi_{0}^{2} \int_{0}^{\infty} d r r^{3}\left\{\left[f^{\prime}(r)\right]^{2}+m_{\chi}^{2}[f(r)]^{2}-\mu_{0}^{2} \theta\left(r-r_{0}\right)[f(r)]^{2}\right\}
$$

We see that if the integral is positive, the value of $\chi_{0}$ which minimizes the action is still $\chi_{0}=0$. On the contrary, if the integral is negative, the action is not bounded from below as $\chi_{0}$ grows.

We choose the following explicit form of $f(r)$ : for $r<r_{0}$ let $f(r)=1$, i.e., $\chi(r)=\chi_{0}$; for 
$r>r_{0}$ let be $f(r)=\exp \left[-\left(r-r_{0}\right) / \delta\right]$. We thus have

$$
\begin{aligned}
S_{\chi} & =\pi^{2} \chi_{0}^{2}\left\{\left(\frac{1}{\delta^{2}}+m_{\chi}^{2}\right) \int_{r_{0}}^{\infty} d r r^{3} e^{-2\left(r-r_{0}\right) / \delta}-\frac{1}{4}\left(\mu_{0}^{2}-m_{\chi}^{2}\right) r_{0}^{4}\right\} \\
& =\pi^{2} \chi_{0}^{2}\left\{\left(\frac{1}{\delta^{2}}+m_{\chi}^{2}\right)\left(\frac{3}{8} \delta^{4}+\frac{3}{4} r_{0} \delta^{3}+\frac{3}{4} r_{0}^{2} \delta^{2}+\frac{1}{2} r_{0}^{3} \delta\right)-\frac{1}{4}\left(\mu_{0}^{2}-m_{\chi}^{2}\right) r_{0}^{4}\right\}
\end{aligned}
$$

It is easy to check that for suitable choices of the parameters the expression within the braces in eq. (18) turns out to be negative. For instance, if $m_{\chi}$ can be disregarded with respect to $\mu_{0}$ and $\delta \sim r_{0}$, the expression is negative provided $\mu_{0} \gg \delta^{-1}$. If $\delta \ll r_{0}$ the expression is negative

provided $\mu_{0} \gg r_{0}^{-1}$, etc. Thus the system is unstable. For the graviton the instability is even worse, because the kinetic term $\tilde{R}^{(2)}$ is not positive-definite.

Physically, we might of course invoke some additional "regularizing" process which comes into play for large values of $\chi_{0}$ and cuts the action. Thus the value of $\chi^{2}(x)$ inside the region is forced by the source to a certain maximum, and this will affect the propagation of the field. The differential equation for the propagator of $\chi(x)$ in the presence of the source must now satisfy additional boundary conditions on each region $\Omega_{i}$. This amounts to a very complex mathematical problem; the physical consequence will be a "damping" of the correlations of $\chi(x)$. One can check this numerically in some model; we shall do this shortly in the next Section, referring to a toy bidimensional spin model.

\section{The formula for the static potential energy.}

In this Section we remind the formula which gives the static potential energy in Euclidean quantum gravity as a functional average [4, 5]. We illustrate its statistical meaning in the case of a weak field through an analogy with a simple 2D Ising model, which we also treat numerically with an elementary simulation. We show numerically that the insertion of local supplementary constraints due to an external field (in analogy to the singularities of the continuum case) damps in a sensible way the spin-spin correlations, and thus the interaction they represent in the model.

Let us first consider, in Euclidean quantum field theory, a scalar field $\Phi(x)$ with action $S_{0}[\Phi]=\int d^{4} x L(\Phi(x))$. In the presence of the external source $J(x)$, the ground state energy of 
the system can be expressed as

$$
\begin{aligned}
E & =\lim _{T \rightarrow \infty}-\frac{\hbar}{T} \log \frac{\int d[\Phi] \exp \left\{-\hbar^{-1}\left[\int d^{4} x L(\Phi(x))+\int d^{4} x \Phi(x) J(x)\right]\right\}}{\int d[\Phi] \exp \left\{-\hbar^{-1} \int d^{4} x L(\Phi(x))\right\}} \\
& =\lim _{T \rightarrow \infty}-\frac{\hbar}{T} \log \left\langle\exp \left\{-\hbar^{-1} \int d^{4} x \Phi(x) J(x)\right\}\right\rangle
\end{aligned}
$$

where it is assumed that the source vanishes outside the interval $(-T / 2, T / 2)$ and that the coupling between $J$ and $\Phi$ is linear. More generally, a formula similar to (19) holds when we are dealing with more fields $\Phi_{A}$ and corresponding sources $J^{A}$, and when the coupling between fields and sources is not linear.

As an useful application of eq. (19), we can write the interaction energy $E(L)$ of two static pointlike sources of the field, kept at a fixed distance $L$. We just need to insert the suitable expression for $J$. The trajectories of the two sources are in flat space

$$
x_{1}^{\mu}\left(t_{1}\right)=\left(t_{1}, 0,0,0\right) ; \quad x_{2}^{\mu}\left(t_{2}\right)=\left(t_{2}, L, 0,0\right) .
$$

In ordinary gauge theories we may re-obtain in this way the Wilson formula for the static quark-antiquark potential. In quantum gravity we are led to the following equation for the static potential

$$
\begin{aligned}
E(L) & =\lim _{T \rightarrow \infty}-\frac{\hbar}{T} \log \frac{\int d[g] \exp \left\{-\hbar^{-1}\left[S[g]+\sum_{i=1,2} m_{i} \int_{-\frac{T}{2}}^{\frac{T}{2}} d t \sqrt{g_{\mu \nu}\left[x_{i}(t)\right] \dot{x}_{i}^{\mu}(t) \dot{x}_{i}^{\nu}(t)}\right]\right\}}{\int d[g] \exp \left\{-\hbar^{-1} S[g]\right\}} \\
& \equiv \lim _{T \rightarrow \infty}-\frac{\hbar}{T} \log \left\langle\exp \left\{-\hbar^{-1} \sum_{i=1,2} m_{i} \int_{-\frac{T}{2}}^{\frac{T}{2}} d s_{i}\right\}\right\rangle_{S}
\end{aligned}
$$

where $S$ is the euclidean action ${ }^{3}$.

In eq.s (21), (22) the lines $x_{1}(t)$ and $x_{2}(t)$ must be parallel with respect to the dynamic metric $g_{\mu \nu}$ and thus they should in principle be re-traced for each field configuration of the functional integral. In practice, it is extremely difficult to compute a functional integral defined in such a formal way. Let us then limit ourselves to consider weak fluctuations of the gravitational field about flat space. The trajectories of the sources may be defined with respect to the background

\footnotetext{
${ }^{3}$ Notice that this formula applies also to two masses which are not pointlike ("pointlike particle" is actually an ill-defined concept in General Relativity), provided we can disregard the internal degrees of freedom. Namely, their action is still equal to $\sum_{i} \int d s_{i}$, where the integrals are taken along the trajectories of the centers of mass.
} 
metric like in (20). It is straightforward to reproduce in this way the Newton potential energy [4, 5]; higher order corrections have been computed too [12]. The geodesic distance between the trajectories $x_{1}(t)$ and $x_{2}(t)$ is now equal to $L$ only on the average; in fact such an approximation is not without physical meaning, since in any realistic source the fixed distance at which the two masses are kept can only be an average value. Also in the non-perturbative evaluations of eq. (22) in quantum Regge gravity [5], the distance $L$ is evaluated a posteriori as the mean value of the geodesic distance on all configurations.

Let us consider the almost-flat metric $g_{\mu \nu}=\delta_{\mu \nu}+\eta_{\mu \nu}$ in a fixed gauge. We obtain

$$
\int_{-T / 2}^{T / 2} d s_{i} \simeq \int_{-T / 2}^{T / 2} d t_{i} \sqrt{1+h_{11}\left[x\left(t_{i}\right)\right]} \simeq T+\frac{1}{2} \int_{-T / 2}^{T / 2} d t_{i} h_{11}\left[x\left(t_{i}\right)\right]
$$

and we see that to a first approximation the fluctuations of $h$ have the effect of making each line "shorter" or "longer". Let us call $\alpha[h]$ the (gauge-invariant) difference between the lenghts of the two lines in a field configuration $h$, and assume for simplicity that the masses of the two sources are equal: $m_{1}=m_{2}=m$. We may expand the exponential in eq. (22), finding (note that $\langle\alpha[h]\rangle$ obviously vanishes by symmetry)

$$
E=2 m+\lim _{T \rightarrow \infty}-\frac{\hbar}{T} \log \left\langle\exp \left\{-\hbar^{-1} m \alpha[g]\right\}\right\rangle=2 m+\lim _{T \rightarrow \infty}-\frac{m^{2}}{2 \hbar T}\left\langle\alpha^{2}[g]\right\rangle+\ldots
$$

This equation exibits an interesting relation between the vacuum fluctuations of the geometry and the static gravitational potential. To illustrate better its "statistical" meaning we would like now to introduce a toy analogy with the 2D Ising model.

Let us consider a planar spin system with periodic boundary conditions and the local coupling $H=-J \sum_{i, j} s_{i j} s_{i^{\prime} j^{\prime}}\left(J>0 ;\left(i^{\prime}, j^{\prime}\right)\right.$ neighbours of $\left.(i, j)\right)$. Let us then consider two columns $j_{1}$ and $j_{2}$ at a distance of $L$ lattice spacings (see fig. 1 ).

We can regard this system as the analogue of a discretized configuration of a $4 \mathrm{D}$ gravitational field on the plane between the two parallel lines of eq. (20). The spin variables \pm 1 represent fluctuations of the metric. At the transition temperature, the fluctuations of the spin variables along the two lines are correlated, approximately like $1 / L$.

Going back to eq. (23) and making the correspondence $s \leftrightarrow h_{11}$, we see that the analogue of $\alpha[g]$ is the difference between spin sums taken along the columns $j_{1}$ and $j_{2}$. The analogue of eq. (24) is

$$
\left\langle\alpha_{\text {Ising }}^{2}\right\rangle=\left\langle\left(\sum_{i} s_{i j_{1}}-\sum_{i} s_{i j_{2}}\right)^{2}\right\rangle=
$$




$$
=\left\langle\left(\sum_{i} s_{i j_{1}}\right)^{2}+\left(\sum_{i} s_{i j_{2}}\right)^{2}-2 \sum_{i} s_{i j_{1}} \sum_{k} s_{k j_{2}}\right\rangle
$$

We are interested only in the term which depends on the distance $L$ between the two columns, that is, the product term

$$
p_{12}=\left\langle\sum_{i} s_{i j_{1}} \sum_{k} s_{k j_{2}}\right\rangle .
$$




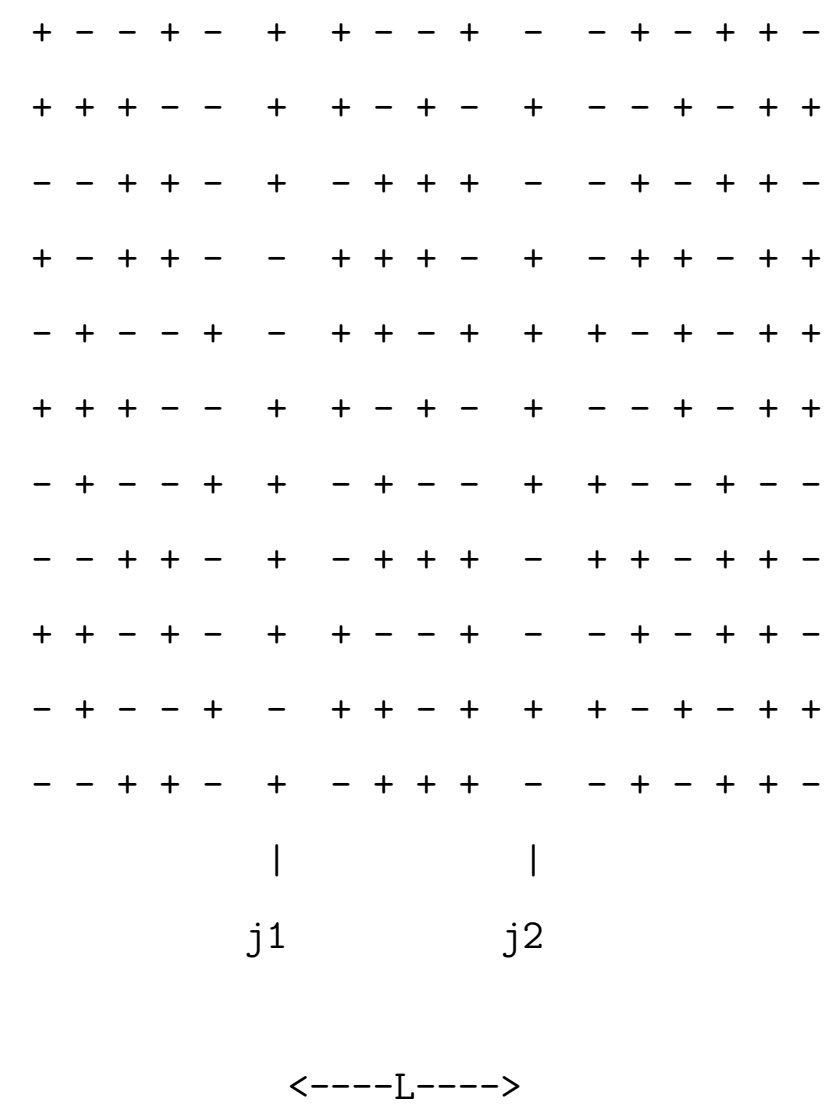

Fig. 1 - Spin sums taken along two columns of a 2D Ising system.

A numerical simulation with a simple $10 \times 12$ system has given, as expected, the following results at the critical temperature:

L (lattice spacings) $\quad p_{-}\{12\}$

\begin{tabular}{|c|c|c|c|}
\hline 2 & 1.15 & $+/-$ & 0.13 \\
\hline 3 & 0.74 & $+/-$ & 0.09 \\
\hline 4 & 0.48 & $+/-$ & 0.09 \\
\hline 5 & 0.42 & $+/-$ & 0.08 \\
\hline 6 & 0.41 & $+/-$ & 0.08 \\
\hline
\end{tabular}

We see that the $L^{-1}$ law is approximately verified also for the correlation between the spin sums taken along the two columns. Only for $L=6$ there is a deviation, which can be explained 
as due to the periodic boundary conditions.

We observe that, due to the nature of the spin system, the correlation between two spin sums along $j_{1}$ and $j_{2}$ is necessarily positive. Thus, the part of $\left\langle\alpha_{I s i n g}^{2}\right\rangle$ which depends on the distance $L$ is negative. In perturbative quantum gravity one finds instead that the correlation $\left\langle h_{11}(x) h_{11}(y)\right\rangle$ is always negative, which leads to the correct negative sign for the potential energy in eq. (24).

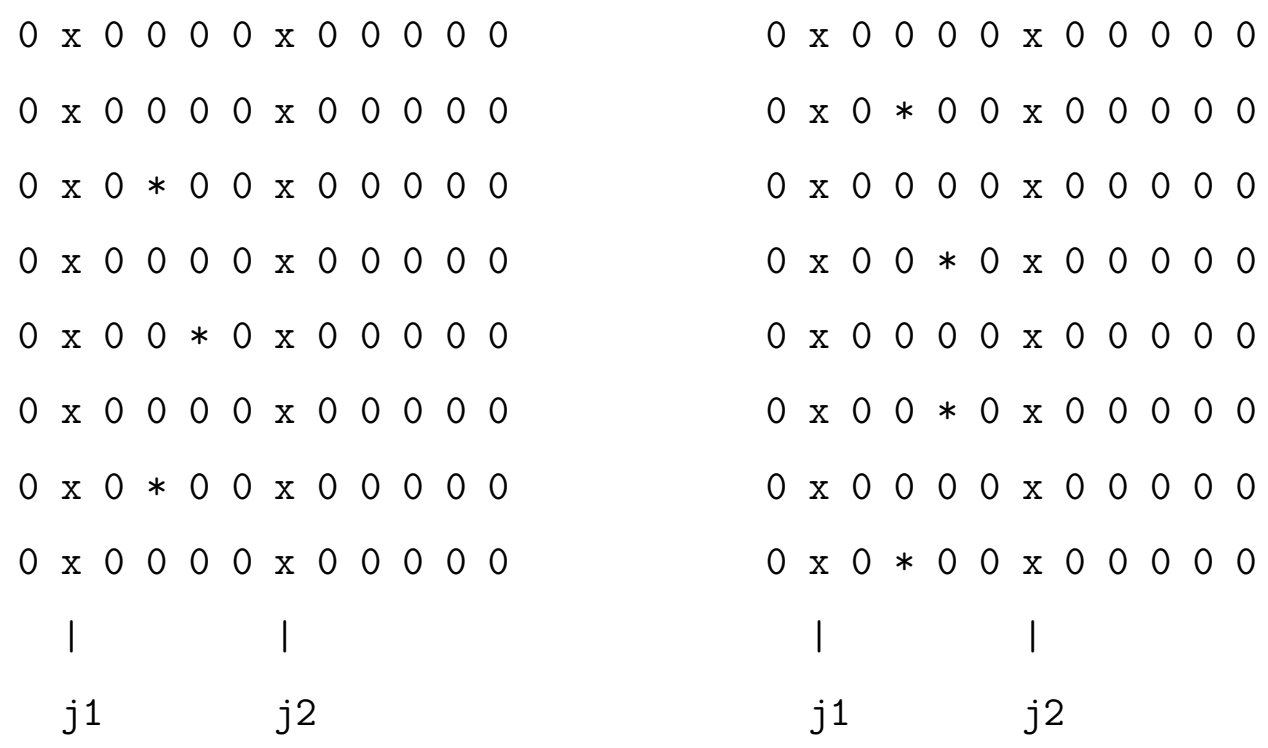

(a)

(b)

\section{Fig. 2 - Insertions of singular points "*" between the columns $\mathrm{j} 1$ and $\mathrm{j} 2$.}

We now introduce some supplementary conditions, in order to simulate the case in which the spin variables assume on certain sites a fixed value. This could be due, like in the gravitational case to which we are interested, to the localized action of an external field. In the spin model we may imagine that an external magnetic field localized on certain sites forces spin-flips. With reference to Fig. 2, let us suppose that the spin-flips occurr on the sites marked with a star and placed between the two columns $j_{1}$ and $j_{2}$ whose correlation we are measuring. (The two columns are denoted by "x", while all the remaining sites are denoted by "0".) To prevent an 
uncontrolled "driving" of the total magnetization, we associate to each spin-flip an opposite flip on a neighboring site. The flips occurr at each Montecarlo step; since the mean frequency at which the regular sites are flipped during the simulation is 120 times smaller, the resulting effect is to force a zero at the "*" sites.

The precise positions of the sites at which the spin-flips happen are almost irrelevant; we find in all cases, as appears from the following table, a sensible diminution of the correlations between the spin sums taken along the columns $j_{1}$ and $j_{2}$ :

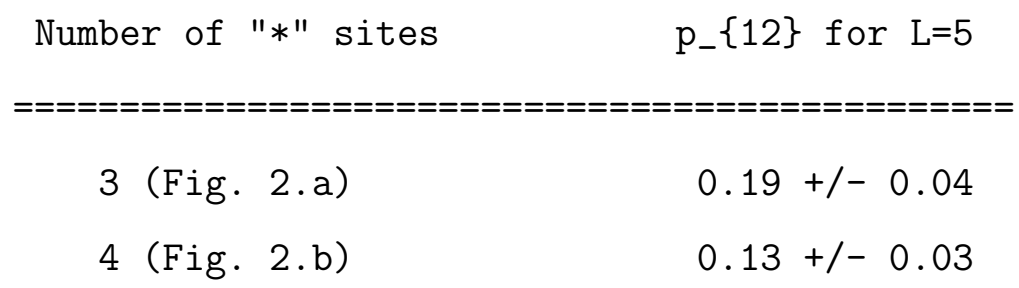

It appears therefore that the insertion of variables which are driven by an external field damps the correlations in the system, and that this mechanism is of a quite general nature, although we are not able to give a precise analytical description yet.

\section{Conclusive remarks.}

We have investigated in this paper an unusual interaction mechanism between gravity and a macroscopic quantum system driven by external fields. This idea was originally suggested by a possible phenomenological application [13, 14], but the mechanism is interesting also from the purely theoretical point of view and deserves further numerical and analytical investigation.

We have shown that under certain conditions the gravitational field becomes unstable and may develope singularities, but we have not tried to find a physical regularization and to compute the effect of the regularized singularities yet. Simple physical analogies show however that they generally reduce the gravitational long-range correlations. Our next task will be the esplicit estimation of the changes in the correlation functions in terms of the squared density $\left|\phi_{0}(x)\right|^{2}$ of the Bose condensate and of its squared gradient $\left[\partial_{\mu} \phi_{0}(x)\right]^{*}\left[\partial^{\mu} \phi_{0}(x)\right]$.

\section{References}


[1] M.J.G. Veltman, in Methods in field theory, Les Houches Summer School 1975, ed.s R. Balian and J. Zinn-Justin, North-Holland, Amsterdam, 1976.

[2] S. Weinberg, Rev. Mod. Phys. 61 (1989) 1; J. Greensite, Phys. Lett. B 291 (1992) 405 and references.

[3] H.W. Hamber and R.M. Williams, Nucl. Phys. B 248 (1984) 392; B 260 (1985) 747; Phys. Lett. B 157 (1985) 368; Nucl. Phys. B 269 (1986) 712. H.W. Hamber, in Les Houches Summer School 1984, Session XLIII, North-Holland, Amsterdam, 1986. H.W. Hamber, Phys. Rev. D 45 (1992) 507; Nucl. Phys. B 400 (1993) 347.

[4] G. Modanese, Phys. Lett. B 325 (1994) 354; Nucl. Phys. B 434 (1995) 697; Riv. Nuovo Cim. 17, n. 8 (1994).

[5] H.W. Hamber and R.M. Williams, Nucl. Phys. B 435 (1995) 361.

[6] L. Garay, Int. J. Math. Phys. A 10 (1995) 145.

[7] C. Rovelli, L. Smolin, Nucl. Phys. B 442 (1995) 593.

[8] G. Modanese, Phys. Lett. B 348 (1995) 51.

[9] N.C. Tsamis and R.P. Woodard, Phys. Lett. B 301 (1993) 351; Comm. Math. Phys. 162 (1994) 217; Ann. Phys. (N.Y.) 238 (1995) 1.

[10] A.D. Dolgov, M.B. Einhorn, V.I. Zakharov, Phys. Rev. D 52 (1995) 717.

[11] H. Van Dam and M. Veltman, Nucl. Phys. B 22 (1970) 397; L.H. Ford and H. Van Dam, Nucl. Phys. B 169 (1980) 126.

[12] I.J. Muzinich, S. Vokos, Phys. Rev. D 52 (1995) 3472.

[13] G. Modanese, Theoretical analysis of a reported weak gravitational shielding effect, report MPI-PhT/95-44, May 1995 (hep-th/9505094); Updating the analysis of Tampere's weak gravitational shielding experiment, report UTF-367/96, Jan 1996 (supr-con/9601001).

[14] E. Podkletnov and R. Nieminen, Physica C 203 (1992) 441; E. Podkletnov and A.D. Levit, Gravitational shielding properties of composite bulk $\mathrm{YBa}_{2} \mathrm{Cu}_{3} \mathrm{O}_{7-x}$ superconductor below 
$70 \mathrm{~K}$ under electro-magnetic field, Tampere University of Technology report MSU-chem, January 1995. 